\title{
The Oligogenic View of Adaptation
}

\author{
G. BELL \\ Department of Biology, McGill University, Montréal, Québec, Canada H3A 181 \\ Correspondence: graham.bell@mcgill.ca
}

\begin{abstract}
The traditional view is that evolution proceeds very slowly, over immense periods of time, driven by weak selection acting on innumerable genes of small effect. Recent studies of rapid evolution, in the laboratory and in the field, have given a radically different picture. Although beneficial mutations tend to be small in effect when they first appear, those that survive to spread and become fixed are usually among the minority with large effect. Hence, although hundreds of loci of small effect may contribute to variation in character state, adaptation is predominantly caused by alleles of large effect. This leads to the hope that the particular mutations responsible for adaptation to altered conditions of life can be identified and characterized. This has been achieved in some cases and may soon become routine. Furthermore, it raises the possibility that adaptive change can be predicted from a knowledge of genetics and ecology. Experimental evolution suggests that any given selection line that is adapting to changed conditions will follow one of a few themes (broadly speaking, loci), each of which may have many variations (mutations within the locus producing similar phenotypes). Hence, evolutionary change can be predicted only within limits, even in principle. Nevertheless, recent attempts to predict how very simple genomes change have been surprisingly successful, and we may be close to a new predictive understanding of the genetic basis of adaptation.
\end{abstract}

History is full of old battles, bitterly contested, leading to an outcome, decisive at the time, which is later quietly reversed. The struggle between saltationist and gradualist views of evolution that spanned the rediscovery of Mendelian genetics is the leading example in our own field. Darwin established the gradualist view at a very early period in the history of evolutionary biology: Natural selection is a weak force, acting on slight inappreciable variation, that leads to adaptation only after the lapse of vast periods of time. Although there were some dissenting voices (including T. Huxley and the codiscoverer A.R. Wallace), this view prevailed up to the debates about the nature of variation around the turn of the 20th century. The convoluted arguments between the biometrical and Mendelian schools have been reviewed by Provine (1971). The outcome was a delicate compromise between the apparently continuous nature of most variation, especially morphological variation, and the discrete hereditary units of Mendelism. It was achieved by supposing that many genes each make a small and equal contribution to the value of most characters, which therefore appear to be very nearly continuously distributed.

The physical basis for this theory lay in a distinction between major genes and "polygenes" (Mather 1941, 1949). Major genes are essential for normal function and development, and mutations are severely deleterious. Polygenes are more or less interchangeable, and the effect of mutation is to cause some trifling alteration of character value. Major genes are responsible for occasional "sports" differing strongly from the ancestral type. Although these figured prominently in early discussions of evolutionary genetics, especially in the Mutation Theory of de Vries

This work is derived in part from previously published material (Bell 2008) by kind permission of Oxford University Press.
(1900), they became discredited as the Mendelian interpretation of continuous variation gained ground. When they were reintroduced by Goldschmidt (1940), the phrase "hopeful monster" became a term of ridicule.

The theoretical interpretation of polygenic variation is the infinitesimal model, which was introduced by Fisher (1918) in the course of his demonstration that Mendelian inheritance necessarily gives rise to the observed pattern of correlation among relatives. Continuous characters are held to be influenced by many genes of small and equal effect, and evolutionary change can be represented in terms of the flux in frequency of these polygenes. This is a mathematical convenience that made it possible to develop Normal theory, in which the mean value of a character can change under selection, while allele frequencies remain almost unchanged. Fisher made it clear, however, that he expected this theoretical extreme to correspond quite closely to the physical basis of variation. His reason is ingenious: Alleles of large effect will be rapidly fixed or lost, leaving only those of small effect segregating in the population. This is unassailable, provided that selection acts consistently in the same direction over long periods of time.

During the half-century following the resolution of the mechanism of inheritance, the gradualist view that adaptation usually involves very slow transformation driven by weak directional selection over long periods of time became firmly entrenched. This had some unfortunate consequences. Field studies were discouraged because they would be unlikely to detect any measurable change within a research program of reasonable duration. Experimental studies of natural selection failed to flourish for the same reason. Although there were some prominent exceptions to these broad statements, the situation began to change decisively only about the time of the Cold Spring Harbor Symposium XXIV in 1959. 


\section{THE BASIS OF QUANTITATIVE VARIATION}

It proved to be very difficult to decide whether quantitative variation is usually attributable to many genes, each with small and nearly equal effects (Mather 1941), or to a few genes of large effect that are de-Mendelized by smalleffect genes or environmental variation (Robertson and Reeve 1952). A number of hypothetical "polygenes" were identified through linkage with major genes, but it was only after 1980 that it was possible to use cheap, highly polymorphic marker systems to locate the genes responsible for quantitative variation routinely and with reasonable precision. They were redubbed "quantitative trait loci," given the acronym QTLs, and thereupon became a fashionable research theme. Identifying QTLs can in principle estimate the number of genes responsible for quantitative variation and the size of their effects, although in practice, these estimates may be biased in several ways (see Erickson et al. 2004). Kearsey and Farquhar (1998) reviewed the literature and found that the average number of QTLs reported was four, with almost all studies reporting eight or fewer. They explained an average of $\sim 50 \%$ of the variance of the character, independently of the number of QTLs. A typical QTL is thus associated with $\sim 10 \%$ of the variance, showing that the variation of quantitative characters is often attributable to a few genes with rather large effects.

In large experiments, the distribution of QTL effects is highly skewed, with a long tail containing a few QTLs of large effect (Edwards et al. 1987 for maize; Mackay 1996 for Drosophila; Hayes and Goddard 2001 for livestock; $\mathrm{Xu} 2003$ for barley). There may be several uninteresting reasons for this (Bost et al. 2001). QTL effects are often reported in terms of the fraction of phenotypic variance explained, which will be proportional to the square of the genetic effect, and the size of effect is biased upward because small studies will only discover large effects (and because studies that fail to detect QTLs might not be published at all). QTLs are not really loci, but rather long and variable chromosome segments between flanking markers; thus, QTLs of large effect might be long segments containing many genes of small effect. Nevertheless, orthologous QTLs are often detected in related species, showing that their effects have been accurately estimated, and the effect of QTLs is unrelated to its length. Effect size can be fitted to negative exponential (Otto and Jones 2000) or $\gamma$ (Xu 2003) distributions. In short, the evidence supports Robertson's view that gene effects will be roughly exponentially distributed, with a few genes of large effect and a much greater number with small effects (Robertson 1967).

\section{THE EFFECT OF BENEFICIAL MUTATIONS}

The genes responsible for adaptation to novel conditions of growth are not necessarily representative of those contributing to the variation of the characters involved. Any character may be influenced, if only to a very small extent, by many genes - most of the genome, perhaps, if sufficiently detailed observations were available. Very few of these are likely to respond to changed conditions of growth. The reason is that a beneficial allele borne by only a few individuals is likely to be lost by drift before increasing to a frequency that will permit it to spread nearly deterministically. It was realized very early in the history of population genetics that the probability of fixation of a novel beneficial mutation is proportional to its selective advantage. Even if the great majority of novel mutations have small effects on fitness, most will soon become extinct. The mutations most likely to be fixed are those of moderate effect, whose rarity is compensated by their greater probability of survival. Consequently, the distribution of effects for the first mutation to be fixed is likely to be modal, with a peak at intermediate values (Kimura 1983).

An elegant interpretation of the initial stages of adaptation can be developed from the assumption that the wild type is likely to be very well adapted, even to conditions that have recently changed. The distribution of effect among all mutations is unknown, but the great majority are deleterious and will not contribute to adaptation. The distribution of effect among the very small minority of beneficial mutations that is alone relevant to adaptation is then predicted from general extreme-value theory, an approach pioneered by Gillespie (1984) and developed more fully by Orr (2003). Suppose that we rank all possible beneficial mutations from 1 (the fittest) to $\lambda$ (the current wild-type allele) and that the difference in fitness between the top-ranked and second-ranked alleles is $\Delta$. Then it can be shown that the average rank of the first mutation fixed is $(\lambda-2) / 4$, and the expected increase in fitness is about $2 \Delta$. Hence, the initial step in adaptation is likely to be the substitution of a beneficial mutation of rather large effect.

This theory predicts that the distribution of the effects of beneficial mutations will shift from exponential when they first appear to modal among those that have become fixed. Kassen and Bataillon (2006) isolated a set of single mutations of Escherichia coli resistant to nalidixic acid and then tested them against the ancestor in medium lacking nalidixic acid. They found that 28/665 mutations increased fitness, and their effects were consistent with an exponential distribution. It is noteworthy that so large a fraction of mutations was beneficial, which reinforces the impression that in some circumstances, beneficial mutation is not very rare. Modal distributions among fixed mutations have been described for E. coli in minimal glucose medium (Rozen et al. 2002) and for Pseudomonas in serine medium (Barrett et al. 2006) by trapping mutations at or near the end of selective sweeps. In the Pseudomonas study, the average effect of a fixed mutation was a doubling of wild-type fitness. These experimental results, although still rather meager, seem to show quite clearly how the predominance of nascent mutations of small effect is translated into fixed mutations of generally much greater effect. It is the latter which imply that selection in novel environments will initially involve large increases in fitness.

The obvious objection to this conclusion is Fisherian: If mutations of large effect are rapidly substituted, only 
polygenes will remain as a source of variation. The response is that conditions change so frequently that a new series of beneficial mutations is continuously recreated. The evidence for this assertion lies beyond this review and is summarized elsewhere (Bell 2008, 2010).

\section{IDENTIFYING THE GENES RESPONSIBLE FOR ADAPTATION}

These theoretical and experimental results suggest that an oligogenic theory of adaptation may often be more appropriate than the infinitesimal model. This is not to suggest that mutation guides the course of evolution, as de Vries (1900) claimed, but rather that adaptation often involves a few discrete steps, each driven by strong selection. One of the most desirable consequences of this view is that the genes contributing to any particular episode of adaptation can be identified, because there are so few of them, and the way in which they are modified can be determined.

A start on this research project was made 30 years ago by a redoutable group of geneticists and biochemists whose work is exemplified in the volume edited by Mortlock (1984). The selection of new amidases in Pseudomonas, for example, is an elegant example of cumulative adaptation to refractory substrates (see Clarke 1984). The ancestral strain could metabolize only the simplest twoand three-carbon amides, acetamide and propionomide. It grows only very slowly on the four-carbon butyramide, because the native amidase is inefficient and is not induced by the new substrate. Adaptation involved the appearance of constitutive mutants that overproduced the amidase, then further modification of gene regulation, and finally a mutation in the amidase structural gene. Further selection led to strains that could grow on more complex amides, including those containing an aromatic ring. There are many similar examples documenting how bacteria adapt to exotic substrates (see Bell 2008). In many cases, the adaptive walk seems to follow a predictable succession of events involving exaptation, deregulation, amplification, and modification (the EDAM model). The particular route toward adaptation is often unique, because of the historical nature of successive substitution. However, it almost always involves a small number of mutations that have a large effect on fitness.

The development of rapid sequencing technology has now made it possible to track evolutionary change in great detail. For a detailed description of the mutations responsible for enhanced glucose uptake after 300 generations of growth in gluose-limited chemostats, see, e.g., Notley-McRobb and Ferenci (1999a,b). In some cases, phosphotransferase activity was elevated, apparently because of loss-of-function mutations at $\mathrm{mlc}$, a gene that regulates sugar transport. The second inner membrane system $\mathrm{Mgl}$ is more important at low glucose concentration and was overexpressed in almost all lines, leading to very large increases in glucose uptake. The underlying genetic changes were substitutions, frameshifts, and short insertions/deletions in both the $m g l$ operator and the MglD repressor protein. At micromolar glucose concentrations, uptake at the outer membrane is undertaken mainly by the LamB glycoporin, which is regulated by mal, which is in turn regulated by the global repressor $m l c$. LamB activity and mal expression were elevated in almost all lines, as the consequence of point mutations in the mal structural gene and by mutations in $m l c$. Thus, the basis of adaptation to glucose-limited chemostat conditions was constitutive production of the LamB protein on the outer membrane and the Mgl proteins on the inner membrane, causing greatly increased uptake of glucose. These mutations lead to an increase in the rate of glucose transport by factors of $8-15$. In glucose-limited chemostats, fitness is linearly related to glucose flux, which will depend primarily on uptake. Consequently, the first beneficial mutations fixed will often have a large effect on fitness. Dykhuizen and Hartl (1981) found that competitive fitness increased by $\sim 13 \%$ in the first 40 generations of culture: This is a minimal estimate of the fitness effect of the first beneficial mutation to be fixed.

For organisms with small genomes, a complete accounting of the genetic basis of adaptation is now feasible. For example, a single lineage of phage $\varphi$ X174 typically adapts to high temperature through mutations in 10-20 nucleotides of the 5400 in its genome. Replicate lines evolve similar levels of fitness through a large number of possible beneficial mutations, some of which are unique to a particular line, whereas others recur in two or more lines. Pairs of replicate lines selected at high temperature on the same host species shared on average $20 \%$ of their beneficial mutations, and $\sim 50 \%$ of all mutations were found in two or more of five replicate lines (Bull et al. 1997). When two replicate lines of $\varphi$ X174 were cultured on a novel host bacterium at high temperature, 22 mutations were fixed, of which 7 occurred in both (Wichman et al. 1999). These shared mutations were substituted in a completely different order in the two populations, however, suggesting that they act independently. In contrast, adaptation of phage T4 to high temperature consistently involved the same point mutations substituted in the same order, and go-back experiments in which stored intermediate sequences were rerun confirmed the repeatability of mutational order (Holder and Bull 2001).

As a very broad-brush conclusion, bacterial or viral evolution in a simple microcosm often seems to involve a few themes and countless variations. The few themes are the major genes where beneficial mutations can occur. The course of adaptation can often be predicted, in terms of the types of genes and proteins likely to be responsible for improvements in growth and fitness, because the number of themes is limited. It cannot be completely predicted, however, because there is usually more than one theme, and this gives rise to genetic differences between lines. The variations are the alleles of the major genes, which may be exceedingly numerous and give rise to genetic diversity within lines. At this level, the course of adaptation is scarcely predictable at all. In the initial stage of adaptation, replicate lines will discover a few broad themes and will then build on these in subsequent evolution.

Nevertheless, the ability to identify the precise mutational steps responsible for adaptation leads to the even more enticing prospect of predicting the course of adapta- 
tion from a knowledge of biochemistry and genetics. For example, most gene expression by phage $\mathrm{T} 7$ requires the RNA polymerase (RNAP) gene. If this is deleted, the equivalent gene from phage T3 can be used, but it is much less efficient. A single base-pair change $(\mathrm{G} \rightarrow \mathrm{C})$ at a particular position $(-11)$ in the $\mathrm{T} 7$ promoter produces a marked elevation in expression. Hence, Bull et al. (2007) predicted that RNAP deletion would be compensated by $\mathrm{G} \rightarrow \mathrm{C}$ mutations at -11 in $\mathrm{T} 7$ promoters when the $\mathrm{T} 3$ gene product was supplied in trans. The outcome was only partly consistent with this prediction. There was indeed a large increase in fitness caused by compensatory mutations, although only about half the promoters were modified. Furthermore, although position -11 was often modified, the most common change was $\mathrm{G} \rightarrow \mathrm{A}$ rather than $\mathrm{G} \rightarrow \mathrm{C}$. Reviewing several similar experiments, Bull and Molineux (2008) concluded that only about one-third to one-half of fixed mutations were successfully predicted, or at least rationalized, from a knowledge of T7 biochemistry. This is scarcely failure: The phage work clearly points the way to a predictive model for population genetics quite different from the black box of polygenes.

\section{ADAPTATION IN NATURAL POPULATIONS}

It has taken half a century for microbial experimental evolution to edge into the mainstream of evolutionary biology, perhaps because many have been reluctant to believe that the relatively simple genomes of phage and bacteria could be used to understand events in complex multicellular organisms. Nevertheless, some of the classical examples of natural selection in the field that began to be systematically investigated in the 1950s, such as industrial melanism in moths and color pattern in snails, were clearly based on the effects of major genes. More recently, mutations in specified major genes have been implicated in other well-studied situations involving continuous or semicontinuous variation.

The three-spined stickleback Gasterosteus aculeatus is a small fish widely distributed in the colder waters of the northern hemisphere. The marine form, living in coastal and brackish water, is heavily armored with dorsal and pelvic spines capable of locking into place and connected by a series of bony plates that extend to the root of the tail. The spines and plates protect sticklebacks from predators by making them difficult to swallow, protecting them from injury, and facilitating their escape (Reimchen 2000). These marine populations have repeatedly invaded streams since the last glacial retreat 10,000-15,000 years ago, giving rise to a very large number of independently derived freshwater populations. Freshwater sticklebacks are often different in appearance from their marine ancestors, and because these differences must have evolved within a few thousand years, or perhaps much less, they have been intensively studied as examples of rapid diversification and even speciation (Hagen and Gilbertson 1973; Bell and Foster 1994; McKinnon and Rundle 2002).

The heavy armor of marine sticklebacks is strongly reduced in populations that have adapted to living in streams. Armor development is controlled largely by a single QTL (Colosimo et al. 2004) that maps to the Ectdysoplasin (Eda) gene (Colosimo et al. 2005). The product of this gene is a signal molecule that is required for normal scale development in other fish and for the development of ectodermal structures such as hair and teeth in mammals. Eda alleles from different populations have common ancestry, suggesting that the invasion of freshwater was accompanied by sorting low-plated alleles from the ancestral marine population. The large pelvic spines of marine sticklebacks are also reduced or completely lost in freshwater populations. Pelvic spine reduction is a Mendelian character involving a single QTL that appears to represent a regulatory mutation in Pitx1, a gene whose homolog is necessary for normal hindlimb development in mice (Shapiro et al. 2004). Plates and spines are reduced very rapidly in freshwater. Bell (2001) cites several cases in which marine populations have moved into newly created or newly vacated freshwater sites and have evolved greatly reduced armor and a deeper body within 10 generations or so. The agent of selection has not been identified, although deep-bodied weakly armored individuals may be more maneuverable and thus more proficient in capturing benthic invertebrates. The strong, repeatable natural selection based primarily on a few genes of large effect echoes the outcome of experimental evolution in laboratory microcosms.

A famous example of an historical process of selection driven by a known selective agent is the change of beak shape in the large ground finch (Darwin's finch) Geospiza fortis on the island of Daphne Major in the Galapagos. A prolonged drought in 1976-1977 caused a change in the composition of the vegetation by favoring plants with large tough-shelled seeds. These could be consumed only by finches with unusually large and powerful beaks, and between 1976 and 1978, beak depth increased at a rate of $26.1 \mathrm{kDar}(0.66 \mathrm{Hal})$ (Boag and Grant 1981). Heavy rain in 1983 reversed the trend in the vegetation by favoring plants with smaller softer seeds that germinated more readily and thereby favored birds with smaller beaks that were more adept at processing them (Gibbs and Grant 1987). Within a few years, the response to reversed selection at a rate of $8.8 \mathrm{kDar}(0.37$ Hal) had more or less restored the status quo (Grant and Grant 1995). This study has become a classic example of selection in a nearly pristine environment, the thoroughness of the fieldwork being buttressed by detailed knowledge of the ecology of the populations and the genetics of beak shape. Beak shape is modulated by Bmp4, whose product is a bone morphogen, which is strongly expressed early in the development of Geospiza species with deep beaks but not in those with long thin beaks (Abzhanov et al. 2004; Grant et al. 2006). Thus, selection on this quantitative character may act primarily through alleles of a single gene to produce adaptation.

A third example is provided by the parallel adaptation of populations of the wood mouse Peromyscus to a dune habitat, principally through the evolution of paler pelage. This involves the fixation of mutations in three genes, apparently in a predictable sequence. This case is described in detail by Hoekstra (2009) in this volume. 


\section{THE OLIGOGENIC VIEW OF ADAPTATION}

One of the most important advances in evolutionary biology since the last CSHL Symposium on evolutionary biology has been the ability to identify and characterize precisely the genetic changes responsible for adaptation. In many cases, it is clear that rapid evolution has been driven by strong selection acting on mutations at one or a few loci. The classical gradualist view that dominated the first century of Darwinism has become supplemented by an oligogenic interpretation that is supported by theory, laboratory experiments, and detailed analyses of selection in natural populations. One welcome outcome of this development is that we are now able to identify precisely the genetic changes that underlie adaptation. It even seems likely that we can begin to build a predictive theory of how populations will evolve in response to some defined stress. This will have very important consequences not only for the study of evolution, but also for our ability to apply evolutionary principles to situations of social and economic concern.

\section{REFERENCES}

Abzhanov A, Protas M, Grant BR, Grant PR, Tabin CJ. 2004. Bmp 4 and morphological variation of beaks in Darwin's finches. Science 305: 1462-1465.

Barrett RDH, MacLean RC, Bell G. 2006. Mutations of intermediate effect are responsible for adaptation in evolving Pseudomonas fluorescens populations. Biol Lett 2: 236-238.

Bell MA. 2001. Lateral plate evolution in the threespine stickleback: Getting nowhere fast. Genetica 112-113: 445-461.

Bell G. 2008. Selection: The mechanism of evolution, 2nd ed. Oxford University Press, Oxford.

Bell G. 2010. Fluctuating selection: The perpetual renewal of adaptation in variable environments. Proc R Soc Lond B Biol Sci (in press).

Bell MA, Foster SA. 1994. The evolutionary biology of the threespine stickleback. Oxford University Press, Oxford.

Boag PT, Grant PR. 1981. Intense natural selection in a population of Darwin's finches (Geospizinae) in the Galapagos. Science 214: 82-85.

Bost B, de Vienne D, Hospital F, Moreas L, Dillmann C. 2001. Genetic and nongenetic bases for the L-shaped distribution of quantitative trait loci effects. Genetics 157: 1773-1787.

Bull JJ, Molineux IJ. 2008. Predicting evolution from genomics: Experimental evolution of bacteriophage T7. Heredity 100: 453-463.

Bull JJ, Badgett MR, Wichman HA, Huelsenbeck JP, Hillis DM, Gulati A, Ho C, Molineux IJ. 1997. Exceptional convergent evolution in a virus. Genetics 147: 1497-1507.

Bull JJ, Springman R, Molineux IJ. 2007. Compensatory evolution in response to a novel RNA polymerase: Orthologous replacement of a central network gene. Mol Biol Evol 24: 900-908.

Clarke PH. 1984. Amidases of Pseudomonas aeruginosa. In Microorganisms as model systems for studying evolution (ed. RP Mortlock), pp. 187-232. Plenum, New York.

Colosimo PF, Peichel CL, Nereng K, Blackman BK, Shapiro MD, Schluter D, Kingsley DM. 2004. The genetic architecture of parallel and armor plate reduction in threespine sticklebacks. PLoS Biol 2: E109.

Colosimo PF, Hosemann KE, Balabhadra S, Villarreal G Jr, Dickson M, Grimwood J, Schmutz J, Myers RM, Schluter D, Kingsley DM. 2005. Widespread parallel evolution in sticklebacks by repeated fixation of Ectodysplasin alleles. Science 307: 1928-1933.

de Vries H. 1900. The mutation theory (transl. JB Farmer and
AD Darbishire). Open Court, Chicago, Illinois.

Dykhuizen DE, Hartl DL. 1981. Evolution of competitive ability in Escherichia coli. Evolution 35: 581-594.

Edwards MD, Stuber CW, Wendel JF. 1987. Molecular-markerfacilitated investigations of quantitative trait loci in maize. I. Numbers, genomic distribution and type of gene action. Genetics 116: 113-125.

Erickson DL, Fenster CB, Stenøien HK, Price D. 2004. Quantitative trait locus analysis and the study of evolutionary processes. Mol Ecol 13: 2505-2522.

Fisher RA. 1918. The correlation between relatives on the supposition of Mendelian inheritance. Trans $R$ Soc Edinb 52: 399-433.

Gibbs HL, Grant PR. 1987. Ecological consequences of an exceptionally strong El Nino event on Darwin's finches. Evolution 68: 1735-1746.

Gillespie JH. 1984. Molecular evolution over the mutational landscape. Evolution 38: 1116-1129.

Goldschmidt R. 1940. The material basis of evolution. Yale University Press, New Haven.

Grant PR, Grant BR. 1995. Predicting microevolutionary responses to directional selection on heritable variation. Evolution 49: 241-251.

Grant PR, Grant BR, Abzhanov A. 2006. A developing paradigm for the development of bird beaks. Biol J Linnean Soc 88: 17 22

Hagen DW, Gilbertson LG. 1973. Selective predation and the intensity of selection acting on the lateral plates of three-spine sticklebacks. Heredity 30: 273-287.

Hayes B, Goddard ME. 2001. The distribution of the effects of genes affecting quantitative traits in livestock. Genet Sel Evol 33: 209-229.

Holder KK, Bull JJ. 2001. Profiles of adaptation in two similar viruses. Genetics 159: 1393-1404.

Kassen R, Bataillon T. 2006. Distribution of fitness effects among beneficial mutations before selection in experimental populations of Pseudomonas fluorescens. Nat Genet 38: 484-488.

Kearsey MJ, Farquhar AGL. 1998. QTL analysis in plants: Where are we now? Heredity 80: 137-142.

Kimura M. 1983. The neutral theory of molecular evolution. Cambridge University Press, Cambridge.

Mackay TFC. 1996. The nature of quantitative genetic variation revisited: Lessons from Drosophila bristles. BioEssays 18: 113-121.

Mather K. 1941. Variation and selection of polygenic characters. J Genet 41: 159-193.

Mather K. 1949. Biometrical genetics. Methuen, London.

McKinnon JS, Rundle HD. 2002. Speciation in nature: The threespine stickleback model systems. Trends Ecol Evol 17: 480-488.

Mortlock RP, Ed. 1984. Microorganisms as model systems for studying evolution. Plenum, New York.

Notley-McRobb L, Ferenci T. 1999a. Adaptive $m g l$-regulatory mutations and genetic diversity evolving in glucose-limited Escherichia coli populations. Environ Microbiol 1: 33-43.

Notley-McRobb L, Ferenci T. 1999b. The generation of multiple co-existing mal-regulatory mutations through polygenic evolution in glucose-limited populations of Escherichia coli. Environ Microbiol 1: 45-52.

Orr HA. 2003. The distribution of fitness effects among beneficial mutations. Genetics 163: 1519-1526.

Otto SP, Jones CD. 2000. Detecting the undetected: Estimating the total number of loci underlying a quantitative trait. Genetics 156: 2093-2017.

Provine WB. 1971. The origins of theoretical population genetics. University of Chicago Press, Chicago, Illinois.

Reimchen TE. 2000. Predator handling failures of lateral plate morphs in Gasterosteus aculeatus: Functional implications for the ancestral plate condition. Behaviour 137: 1081-1096.

Robertson A. 1967. The nature of quantitative genetic variation. In Heritage from Mendel (ed. A Brink), pp. 265-280. University of Wisconsin Press, Madison.

Robertson FW, Reeve E. 1952. Studies in quantitative inheri- 
tance. I. Effects of selection of wing and thorax length in Drosophila melanogaster. J Genet 50: 414-448.

Rozen D, de Visser JA, Gerrish P. 2002. Fitness effects of fixed beneficial mutations in microbial populations. Curr Biol 12: 1040-1045.

Shapiro MD, Marks ME, Peichel CL, Blackman BK, Nereng KS, Jónsson B, Schluter D, Kingsley DM. 2004. Genetic and developmental basis of evolutionary pelvic reduction in threespine sticklebacks. Nature 428: 717-723.

Wichman HA, Badgett MR, Scott LA, Boulianne CM, Bull JJ. 1999. Different trajectories of parallel evolution during viral adaptation. Science 285: 422-424.

Xu S. 2003. Estimating polygenic effects using markers of the entire genome. Genetics 163: 789-801. 


\section{$8_{8}^{\infty} \mathrm{CSH} \&$ Cold Spring Harbor Symposia SYMPOSIA on Quantitative Biology}

\section{The Oligogenic View of Adaptation}

G. Bell

Cold Spring Harb Symp Quant Biol 2009 74: 139-144 originally published online August 10, 2009 Access the most recent version at doi:10.1101/sqb.2009.74.003

References This article cites 36 articles, 4 of which can be accessed free at: http://symposium.cshlp.org/content/74/139.full.html\#ref-list-1

License

Email Alerting Receive free email alerts when new articles cite this article - sign up in the box at the Service top right corner of the article or click here.

To subscribe to Cold Spring Harbor Symposia on Quantitative Biology go to:

http://symposium.cshlp.org/subscriptions 